\title{
Coherent variability of GX 1+4
}

\author{
Ann-Sofie Bak Nielsen ${ }^{1 \star}$ and Alessandro Patruno ${ }^{1,2}$ \\ ${ }^{1}$ Leiden Observatory, University of Leiden, Niels Bohrweg 2, NL-2333 CA, Leiden, the Netherlands \\ ${ }^{2}$ ASTRON, the Netherlands Institute for Radio Astronomy, Postbus 2, NL-7900 AA, Dwingeloo, the Netherlands
}

Accepted 2018 June 6. Received 2018 May 25; in original form 2018 March 7

\begin{abstract}
The accreting pulsar GX $1+4$ is a symbiotic X-ray binary system with an M-type giant star companion. The system has a spin period of about $150 \mathrm{~s}$ and a proposed strong magnetic field of $10^{12}-10^{14} \mathrm{G}$. In this paper we study the coherent variability of the source and attempt to find a phase-coherent solution for the pulsar. We also test for the presence of a pulse phase-flux correlation, similar to what is observed for the accreting millisecond X-ray pulsars, in order to test whether this feature is dependent on the magnetic field strength. We find that no phase coherent solution exists, which suggests that the pulsar is accreting plasma from a wind rather than an accretion disc. We also find evidence that the pulse phase is not correlated with the Xray flux, which strengthens the idea that such relation might be present only in weak magnetic field sources like accreting millisecond pulsars.
\end{abstract}

Key words: binaries: symbiotic - stars: neutron - pulsars: individual: GX 1+4-X-rays: binaries.

\section{INTRODUCTION}

The X-ray pulsar GX 1+4 was first discovered in 1970 by balloon $\mathrm{X}$-ray observations at energies above $15 \mathrm{keV}$ by Lewin, Ricker \& McClintock (1971). GX 1+4 is the first discovered accreting pulsar in a low-mass symbiotic X-ray binary system (SyXB) and it is unique among the symbiotic binaries, due to the primary star being a neutron star and not a white dwarf or a main sequence star (Davidsen, Malina \& Bowyer 1977; Hinkle et al. 2006; Serim et al. 2017). The companion of GX $1+4$ is V2116 Oph (Chakrabarty et al. 1997), which is an $\mathrm{M}$ giant star, of about $1.2 \mathrm{M}_{\odot}$ and the pulsar is thought to have a mass of $1.35 \mathrm{M}_{\odot}$ (González-Galán et al. 2012). The M giant is probably not filling its Roche Lobe, but the pulsar is instead accreting through a stellar wind (Chakrabarty et al. 1997; Hinkle et al. 2006). The orbital period of GX $1+4$ was originally proposed to be 304 d (Cutler, Dennis \& Dolan 1986; Pereira, Braga \& Jablonski 1999). This value was, however, revised to be $1161 \mathrm{~d}$ and it remains to be confirmed (Hinkle et al. 2006). The orbit is modestly eccentric with $e 0.10$ (Hinkle et al. 2006). Torque reversals are observed in GX 1+4, with the first observations showing a clear neutron star spin-up (Nagase 1989) that, around 1987, when the pulsar reappeared, turned into a clear spin-down. During the spin-up phase, the pulsar had a spin frequency derivative of $\dot{v}=$ $6.0 \times 10^{-12} \mathrm{~Hz} \mathrm{~s}^{-1}$, whereas during spin-down the pulsar had a spin frequency derivative of $\dot{v}=-3.7 \times 10^{-12} \mathrm{~Hz} \mathrm{~s}^{-1}$ (Chakrabarty et al. 1997). The spin period was about $159.9 \mathrm{~s}$ in 2010 (Yoshida et al. 2017).
${ }^{13-14}$ Rea et al. (2005) observed a possible electron cyclotron feature in the energy spectra, using BeppoSAX, at an energy of 32-37 keV. This would correspond to a magnetic field of about $4 \times 10^{12} \mathrm{G}$, which is lower than earlier magnetic field estimates of order $B \sim 10 \mathrm{G}$ (Dotani et al. 1989; Mony et al. 1991; Cui \& Smith 2004), inferred from spin-down episodes. It must be remarked, however, that the proposed identification of the cyclotron line is still unconfirmed (Rea et al. 2005).

An open question about GX $1+4$, as well as other slow-accreting pulsars in low-mass X-ray binaries, is whether the presence of the neutron star magnetic field and its interaction with the accretion flow could lead to phenomena similar to those observed in accreting millisecond X-ray pulsars (AMXPs), which are much fainter and with lower magnetic fields (see Patruno \& Watts 2012 for a review). The reason is that observing a similar phenomenology, for example, in the behaviour of pulsations might shed some light on how accretion flows and neutron star magnetospheres interact and clarify the exact role of the strength of the magnetic field and mass accretion rate. For example, a still unexplained correlation between the X-ray flux and the pulse phases is observed in several AMXPs (Patruno, Wijnands \& van der Klis 2009), contrary to what is predicted by accretion torque theory, where it is the spin frequency derivative and not the pulse phase that is expected to be correlated with the X-ray flux. Iłkiewicz, Mikołajewska \& Monard (2017) discussed the variability of GX $1+4$, reporting large variations in the $\mathrm{X}$-ray flux over several energy bands. The variations reach up to one to two orders of magnitude (Iłkiewicz et al. 2017; Serim et al. 2017). Due to these large variations in X-ray flux, we consider GX $1+4$ an optimal system to test whether corresponding large variations in the pulse phases are present. Patruno et al. (2009) examined 
six known AMXPs based on the presence of red timing noise in the pulse phases. They found that there was a correlation and, in some cases, an anticorrelation, between the flux and pulse phase. This was interpreted as due to the motion of the hot spot on the surface of the pulsar in response to mass accretion rate variations. It is currently not known whether a similar phenomenon exists in high field pulsars since the stronger magnetic field might prevent the movement of plasma on the neutron star surface. Bak Nielsen, Patruno \& D'Angelo (2017) and Patruno et al. (2012) found that such phase-flux correlation was not present in two moderately high field accreting pulsars, namely 2A $1822-371$ and Terzan 5 X-2. Despite the lack of phase-flux correlations, some timing noise was however observed in the pulse phase residuals of these systems. Furthermore, the strength of their magnetic fields is only 1-2 orders of magnitude larger than what is seen in AMXPs, whereas their accretion rate is close to the Eddington limit. Therefore it remains to be verified whether higher magnetic field accreting pulsars show such correlation or not. Testing this hypothesis is very valuable for two reasons. On the one hand, if the hot spot is moving on the surface, then this effect must be taken into account when finding a pulsar timing solution. Second, the magnetic field of GX $1+4$ is four to six orders of magnitude larger than those found in AMXPs and thus detecting (or not detecting), this phenomenon will help understand its origin.

In this paper we examine long- and short-term fluctuations of the pulse time of arrivals and variations of the X-ray flux and we check whether there is any evidence for a pulse phase-flux correlation on the prototype system GX 1+4. In Section 2 we go through the observations used, and in Section 3 we present the results of our analysis. In Section 4 we discuss the possible presence (or lack thereof) of a pulse phase-flux correlation and the observed short-term variability of the source and we provide a physical interpretation of what is observed.

\section{OBSERVATIONS}

We have used data taken between 2001 March 6 and November 14 (ObsID = 60060), thus using almost the same data range as Serim et al. (2017). We choose this data set because Serim et al. (2017) claim to have phase connected the data and provide a timing solution for the source. The data were recorded with the Proportional Counter Array (PCA), which was on board of the Rossi X-ray Timing Explorer (RXTE). RXTE/PCA has five xenon/methane proportional counter units that are sensitive in the energy range of 2-60 keV (Jahoda et al. 2006). We use the event files with a resolution of $2^{-20} \mathrm{~s}$ (GoodXenon) for the timing analysis, and the Standard-2 data-mode, with a $16 \mathrm{~s}$ time resolution, to create the X-ray light curve. The light curve is created in the $2-16 \mathrm{keV}$ energy range and the X-ray flux is averaged for each observation (ObsID) and normalized in Crab units (see the top panel in Fig.1). A detailed description of this procedure can be found in van Straaten, van der Klis \& Méndez (2003). We perform the timing analysis by selecting the energy channels 9-67, which correspond to an energy range of about 3-20 keV, as specified by Serim et al. (2017). The data were barycentered using the FTOOL faxbary, using the JPL D405 Solar System coordinates and the source coordinates, from Cutri et al. (2003), RA: 17:32:02.16, DEC:-24:44:44.2. We then epoch folded the barycentered data in pulse profiles of 32 bins over the spin period, $\mathrm{P}_{\mathrm{s}}=136.3228 \mathrm{~s}$ for segments of length $\sim 2000$ 3500 s. We cross-correlated each pulsation with a sinusoid at the spin frequency and generated the time of arrivals (TOAs) for this data set. We selected only pulsations with a signal-to-noise $(\mathrm{S} / \mathrm{N})$ larger than $3.3 \sigma$ in order to achieve less than one false detection in our ensemble (composed by a total of $N=538$ pulse profiles). The $\mathrm{S} / \mathrm{N}$ is defined as the ratio between the pulse amplitude and its $1 \sigma$ statistical error. The ephemeris used during the epoch folding is taken from Serim et al. (2017). To phase connect the data, we used TEMPO2 version 2012.6.1.

\section{RESULTS}

In the following sections we will go though the flux variations of GX $1+4$ and compare these to variations in the pulse phase, in order to test if there is a correlation or anticorrelation. We look at both the full data set and three small segments of the data.

\subsection{Phase connection}

We have used archival data from RXTE approximately corresponding to the data segment labelled ' $a$ ' in Serim et al. (2017), and we used the coherent timing solution from the same paper (see Table 1). We do this in order to reproduce their results and inspect the pulse phase residuals in order to later look for a pulse-phase correlation in the data.

When folding the data we clearly detect pulsations with high S/N, but the pulse phase residuals are scattered between -0.5 and +0.5 pulse phase cycles, which means that the pulsations are not phase connected. We thus cannot recover the same results of Serim et al. (2017) with their proposed timing solution.

We thus consider the possibility that the true spin frequency (and derivatives) of GX 1+4 might be off with respect to the solution proposed by Serim et al. (2017). To look for a phase connected solution, we thus keep all parameters fixed and vary the pulse frequency to explore the $\chi^{2}$ surface and select the value that minimizes the $\chi^{2}$. We used 1000 different pulse frequencies, varying between $0.0071355 \mathrm{~Hz}$ and $0.0075355 \mathrm{~Hz}$ in steps of $4 \times 10^{-7}$ $\mathrm{Hz}$. The pulse frequency corresponding to the minimum $\chi^{2}$ found still did not give a phase-connected solution. We then considered also variations of the pulse frequency derivative $\dot{v}$, exploring values between $-2.24585 \times 10^{-12} \mathrm{~Hz} \mathrm{~s}^{-1}$ and $-1.6585 \times 10^{-12} \mathrm{~Hz} \mathrm{~s}^{-1}$ in steps of $8 \times 10^{-16} \mathrm{~Hz} \mathrm{~s}^{-1}$ and again we were unable to find a phaseconnected solution. To further investigate the reason of this, we considered also the presence of a $\ddot{v}$, since Serim et al. (2017) provide also a measured value for that parameter. However, we found no improvement in our attempt to phase connect the data. This is not surprising since the contribution of $\ddot{v}$ to the pulse phase variation with time can be considered negligible when compared to $v$ and $\dot{v}$. A difference between our analysis and that of Serim et al. (2017) is that our data set spans a time range slightly larger than theirs, by about 2 months. However, again this makes no difference with regard to the final solution since the phase connection is not achieved in any segment of the data. We do see pulsations with high signal-to-noise ratio throughout the observations, which means that the solution is sufficiently accurate to fold the data. An example of the pulse phase residuals found when using, for example, the solution given in Table1 is given in Fig. 1. It should, however, be noted that on fig. 2 (upper panel) of Serim et al. (2017), the phase residual has a variation greater than 1 cycle, therefore the timing solution used in the cross-correlation analysis of pulse profiles with template pulse leading to a scatter between -0.5 and 0.5 , makes it impossible to recover their results on long time-scales. However, using the method by Serim et al. (2017), this timing residuals can be phase connected, in so far as phase connected means a solution that accounts for all signal and noise, if a high degree polynomial 

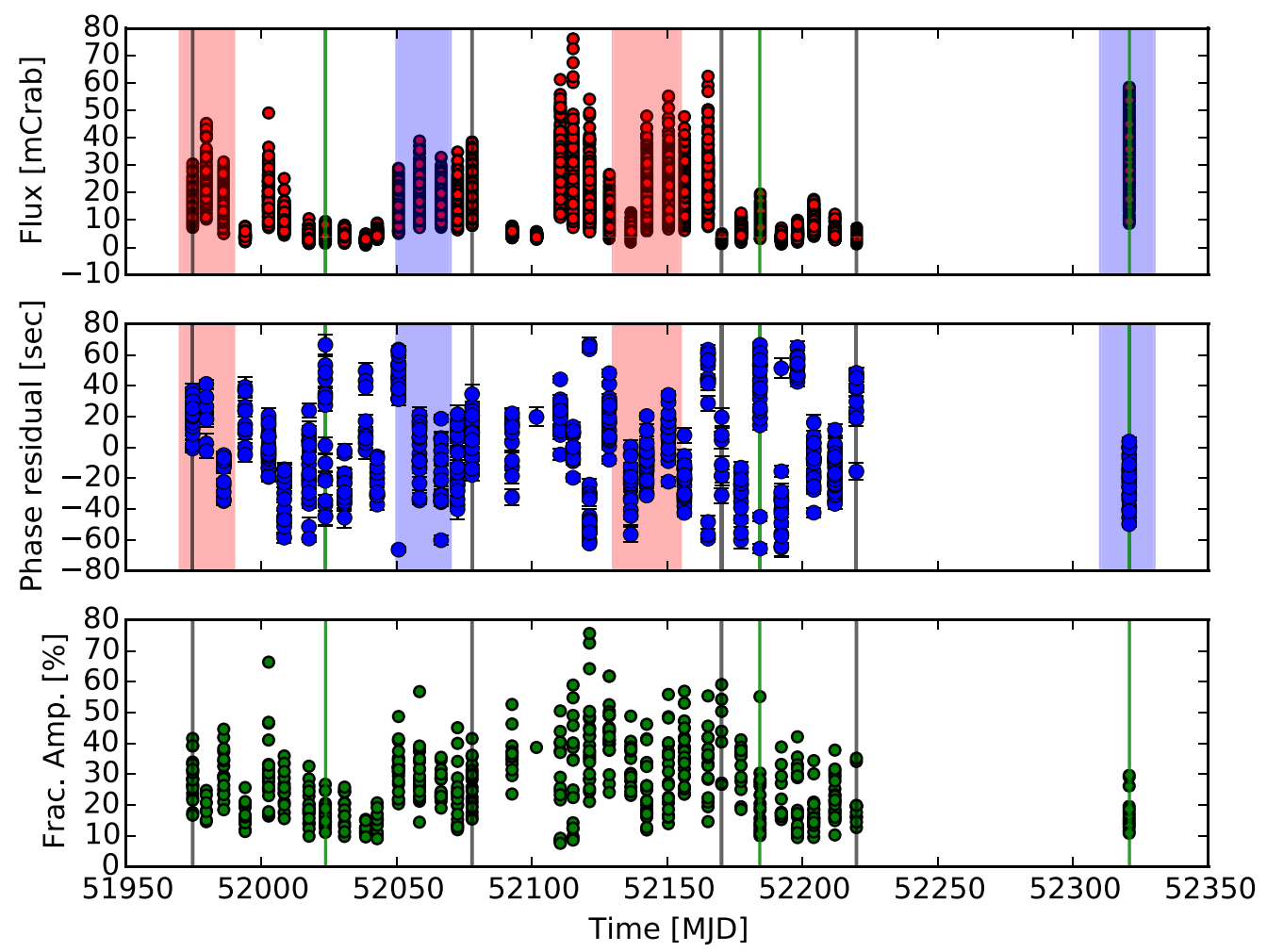

Figure 1. Top panel: The light curve for the RXTEdata between 2001 March 6 and November 14. Lower panel: The corresponding pulse phase. The red area marks the correlations between the flux and pulse phase, the blue area marks the anticorrelation, and the green line marks the data segment that is zoomed in on in Fig. 2. The black lines mark the 4 times for which pulse profiles are shown in Fig. 3.

Table 1. The parameters used in the epoch folding.

\begin{tabular}{lr}
\hline Parameter & Value \\
\hline MJD & $51974.7-52320.7$ \\
RA & $17: 32: 02.16$ \\
DEC & $-24: 44: 44.2$ \\
Epoch & 51974.0 \\
$v$ & 0.007335526 \\
$\dot{v}$ & $-2.0585 \times 10^{-12}$ \\
\hline
\end{tabular}

is fitted. In this paper, we use coherency to describe a solution that does not only account for the signal and noise in the time window of the observations (since it is always possible to fit $\mathrm{N}$ data points with a polynomial of order $\mathrm{N}-1$ at most), but is also able to predict the future pulse phases.

Finally, since we are using only the fundamental frequency for our coherent timing analysis, we tested the presence of a second and third harmonic in the pulse profiles. Both were present in the data with relatively high significance ( $\mathrm{S} / \mathrm{N}$ up to $\sim 15$ ). However, they did not behave differently from the first harmonic (i.e. the fundamental), and no phase-connected solution could be found in this case.

\subsection{Phase-flux correlation}

Another way to search for a phase-connected solution is to try to maximize the strength of the phase-flux correlation, which has been observed so far in several accreting millisecond pulsars (for a more detailed discussion of this method, see for example Patruno 2010). We thus tested whether there are similarities in the variations of the flux with the pulse phase residuals as seen in Patruno et al. (2009). To obtain the exact count rate, we used only one PCU, namely PCU2, since this unit is always on during all observations. We use the count rate in this case because in this way we can create a light curve with the exact same time binning as the pulse phase time series. We then kept the Keplerian orbit fixed (see Table 1) and varied the pulse frequency and frequency derivative as explained in the previous section. We then fitted the data with the linear correlation

$\phi=a+b F_{x}$

where $\phi$ is the pulse phase, $F_{\mathrm{x}}$ is the X-ray count-rate, and 'a' and ' $b$ ' are fitting parameters. We explored the $\chi^{2}$ surface to find the value of $v$ that minimizes the $\chi^{2}$ value. We explored a grid of 1000 values for $v$ and then fitted the phase residuals versus flux with the linear relation in equation (1). We then found the minimum rootmean-squared (rms) value of the fit that gave us the best fit value of $v$ $\sim 0.007417326 \mathrm{~Hz}$. We re-folded the data with the new value for $v$ and iterated this entire procedure for a few times, but the minimum $\chi^{2}$ value did not converge. This means that we are unable to find a global $\chi^{2}$ minimum corresponding to a phase-connected solution of the data. We then repeated the entire procedure by adding also the pulse frequency derivative $\dot{v}$ (and using again 1000 guess values) to verify whether the lack of a phase connection could be ascribed to a rapid variation of the pulse frequency over the time span of the observations. However, we could not find a global minimum for the $\chi^{2}$ even in this case.

In summary, when using both standard $\chi^{2}$ minimization methods with the pulse phases alone and when using the pulse-phase correlation method, we cannot phase connect the solution. We stress, however, that pulsations were detected throughout the observations, 

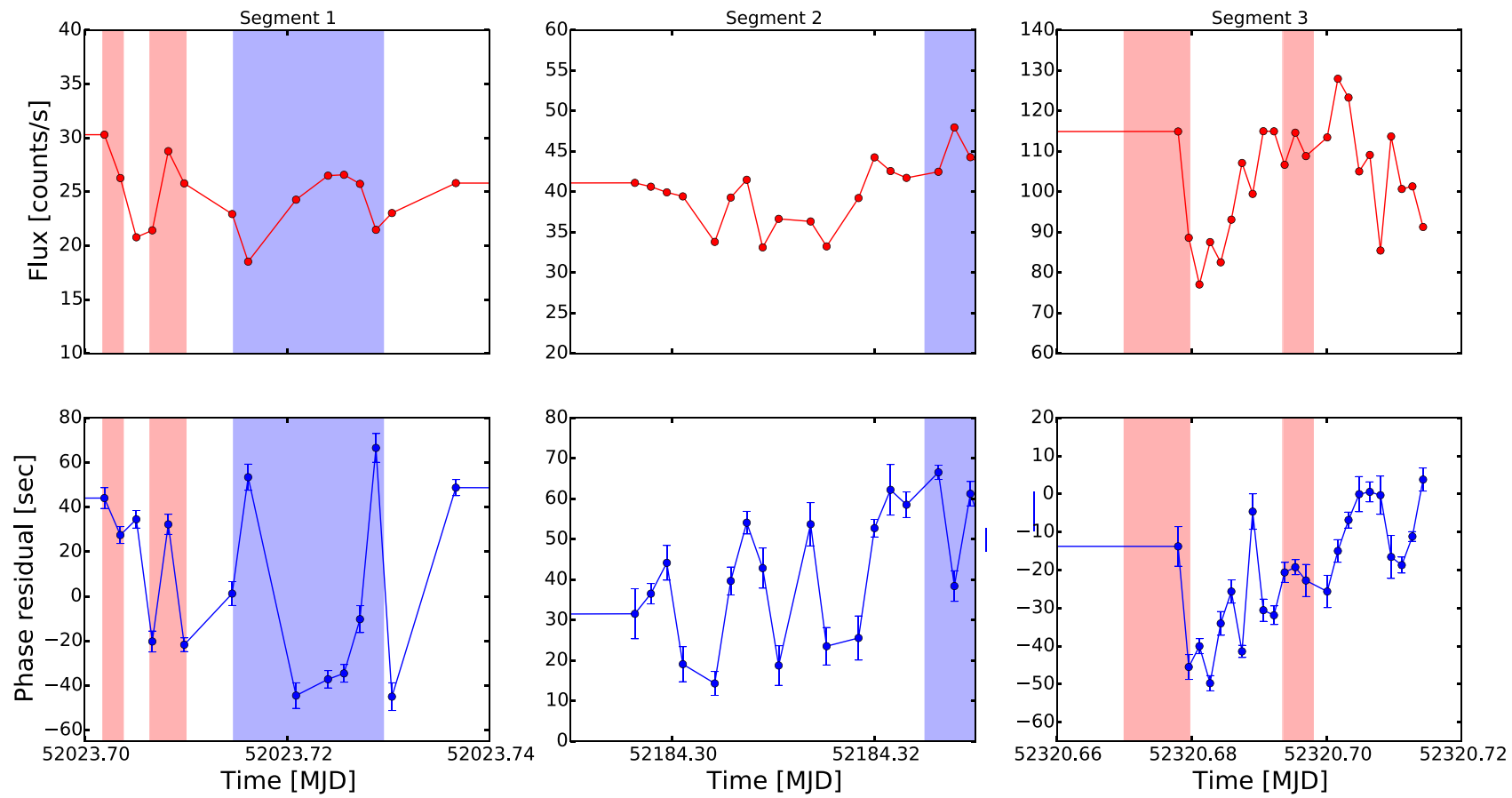

Figure 2. Top panel: Light curve of small data segment. Lower panel: Pulse phase residuals for the same data segment. The data segment is marked on Fig.1 in green. The red areas mark the correlations between pulse phase and flux and the blue are marks the anticorrelation between pulse phase and flux.

which means that a coherent signal is present in the data, at least on a short time-scale (of the order of a few rotational periods of the pulsar). The coherence of the signal is then lost when looking at longer (> hours) time-scales. We then went back and used the TOA solution given in Table 1 (Serim et al. 2017), and inspected the resulting light curves and pulse phase residuals, showing the minimum rms. These are shown in Fig. 1. The reason for this choice is that if the pulse phases keep their coherence on short time-scales, then it should still be possible to see correlations between flux and pulse phases on these time-scales (assuming that such an effect exists in GX 1+4). From the figure it is clear that there is no simple long-term correlation or shape that is similar in the top two panels, but there are indeed a few segments of the top two panels on Fig. 1, where the flux and pulse phase residuals are correlated or anticorrelated. These are, respectively, marked in blue (anticorrelation) and red (correlation). The green lines on Fig. 1 are the three sections that are zoomed in on in Fig. 2, and the grey lines are the times where the pulse profiles are plotted, as seen in Fig. 3.

\subsection{Short-term variability}

Since we do see pulsations in every data point plotted in Fig. 1, this means that we recover part of the coherent signal with the solution used. On Fig. 2, the three data segments marked in green (in Fig. 1) are shown. The three segments were chosen due to the variations in the flux and phase residuals, where segment 1 has almost no variation in the flux but a large variation in the phase residuals, segment 2 has small variations in the flux with larger variations in the phase residuals but not as large as segment 1 , and the third segment has a variation in both flux and phase of somewhat similar order. On Fig. 2 the average of the phase residuals is corresponding to an overall slope of zero, indicating that the frequency is correctly measured. When looking at Fig. 2, it is evident that there is no clear correlation between the flux and phase residuals on a short time-scale.
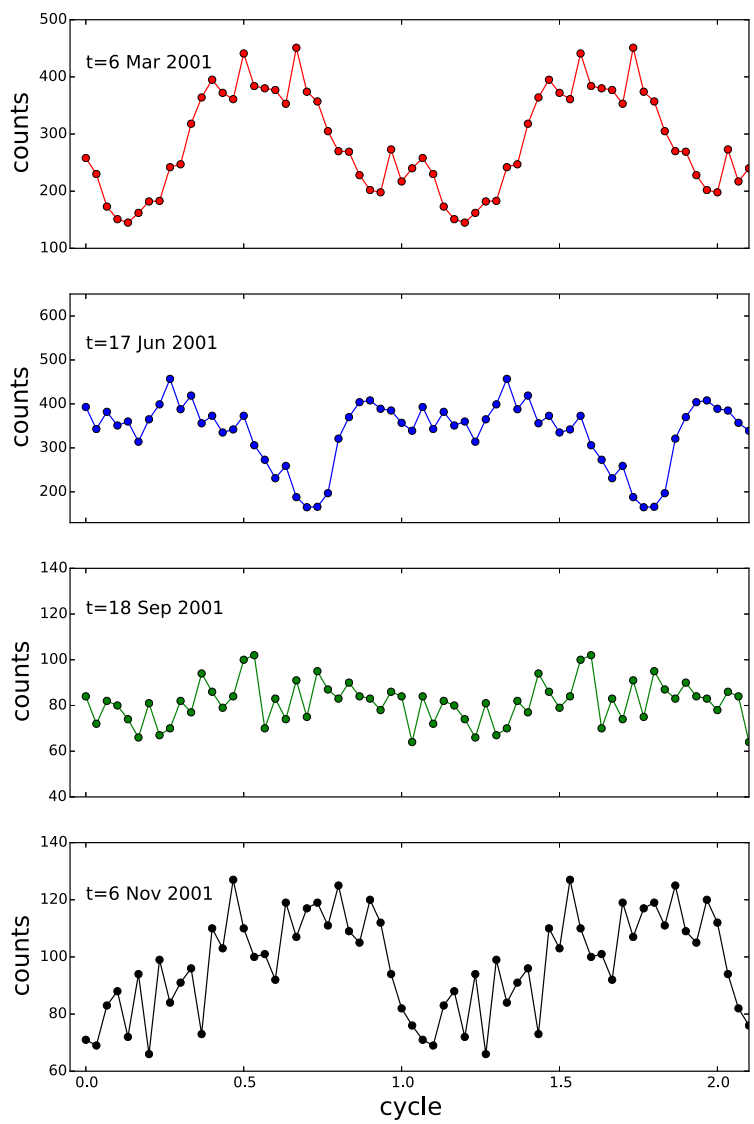

Figure 3. Four pulse profiles at different times. The first and last pulse profiles in the figure correspond to the initial and final epoch of the folded data. The two middle panels correspond to in-between times (marked in each panel). 


\section{DISCUSSION}

\subsection{Pulse profiles}

The TOA solution we have used throughout this paper, found by Serim et al. (2017), was found using a template pulse profile. As reported earlier by Cui \& Smith (2004), and as shown on Fig. 3, we see that the pulse profiles vary throughout the observation, so that it is not possible to use a single template high signal-to-noise pulse profile to perform the coherent timing analysis. Indeed, when the pulse shape variability is not properly taken into account, there is the risk of introducing strong systematics in the results (Boynton et al. 1984; Hartman et al. 2008; Ibragimov \& Poutanen 2009). We have, however, used the solution found by Serim et al. (2017) as our guess solution in order to find a phase-connected solution of each harmonic separately (up to the third harmonic), so as to avoid the ambiguity in the definition of the pulse phase reference point. However, despite this precaution, we find that it is not possible to create a phase-connected solution. The pulse profiles seen on Fig.3 show an example of the variability in the pulse shape.

\subsection{Phase-flux correlation - consequence for AMXPs}

A proposed scenario for explaining the pulse phase and flux correlation in accreting millisecond pulsars was the movement of the hot spot, perhaps linked to movement of the magnetic field ( $\mathrm{Pa}$ truno et al. 2009). In this paper and in the paper by Bak Nielsen et al. (2017), it has been tested if there is any such correlation in the pulsars with a higher magnetic field, which would possibly rule out movement of the hot spot. It is found in this paper and in Bak Nielsen et al. (2017), concerning the LMXB pulsar 2A 1822-371, that it is not possible to find a phase-flux correlation. As described in Section 3.2 in this paper, it is also not possible to find any phaseflux correlation for GX 1+4. Because 2A 1822-371 and GX 1+4 are pulsars with a higher magnetic field than the AMXPs, it is possible that the pulse phase-flux correlation found in AMXPs (Patruno et al. 2009) is dependent on the magnetic field strength. If the magnetic field is strong enough, this could prevent the movement of the hot spot on the neutron star surface, which was suggested to be a possible explanation for the phase-flux correlation (Patruno et al. 2009).

\subsection{Short-term flux variability}

We can infer, from our attempts to phase connect the data, that the signal from GX $1+4$ is not coherent over a time-scale of days or longer. Furthermore, since we see short-term variability in both the flux and the phase and we do not see a correlation between the flux and phase, we infer that something is varying quickly on the neutron star surface. This is also supported by the change in the pulse profile shape. The variation could, for example, be the shape of the hot spot or perhaps the geometry of the beam. We observe pulsations throughout the observations, which does imply that even though we are not able to find a phase-connected solution, we keep the coherence of the signal on a time-scale of hours.

From the above, we would infer that wind accretion is quite likely. The wind would attach to random field lines and thus create shortterm variability and pulse shape variability, and make it impossible to find a phase coherent (long-term) solution (González-Galán et al. 2012; van den Eijnden et al. 2018). The short-term variability is similar to variability seen in Vela X-1, an HMXB, that accretes through wind accretion (Boynton et al. 1986; Malacaria et al. 2016).
However, it should be noted that it is, at least on short times-cales, possible to create coherent timing solutions to Vela X-1 (Nagase 1989). Due to the short-term variations, wind accretion seems to be the most likely accretion mechanism of GX $1+4$, considering the variability on short time-scales.

\section{CONCLUSIONS}

We examined about a year of RXTEdata of GX 1+4, from 2001 March to 2002 February. We conclude that it is not possible to phase connect the data due to the nature of the accretion process, which most likely proceeds via wind from the giant donor companion in the binary. We tested if a pulse phase-flux correlation was present in the data, similar to what is sometimes seen in the AMXPs and find that such a correlation does not appear to be present in GX $1+4$. This might be due to the stronger magnetic field of the pulsar or, alternatively, to the different nature of the accretion process, which, in AMXPs, proceeds via an accretion disc interacting with the pulsar magnetosphere. The findings of this paper, along with the findings of Bak Nielsen et al. (2017), do suggest that the phase-flux correlation is present only for pulsars with low magnetic fields of the order $10^{8}-10^{9} \mathrm{G}$, as is the case for the AMXP. In this paper, we further suggest that there are some similarities between GX 1+4 and HMXBs such as for example VELA X-1; they both show variations in the pulse profile that is suggested to originate from wind accretion.

\section{ACKNOWLEDGEMENTS}

ASBN and AP acknowledge support from a NWO Vidi Fellowship. The authors would like to thank the referee for useful comments.

\section{REFERENCES}

Bak Nielsen A.-S., Patruno A., D’Angelo C., 2017, MNRAS, 468, 824 Boynton P. E., Deeter J. E., Lamb F. K., Zylstra G., Pravdo S. H., White N. E., Wood K. S., Yentis D. J., 1984, ApJ, 283, L53

Boynton P. E., Deeter J. E., Lamb F. K., Zylstra G., 1986, ApJ, 307, 545

Chakrabarty D. et al., 1997, ApJ, 481, L101

Cui W., Smith B., 2004, ApJ, 602, 320

Cutler E. P., Dennis B. R., Dolan J. F., 1986, ApJ, 300, 551

Cutri R. M. et al., 2003, VizieR Online Data Catalog, 2246

Davidsen A., Malina R., Bowyer S., 1977, ApJ, 211, 866

Dotani T., Kii T., Nagase F., Makishima K., Ohashi T., Sakao T., Koyama K., Tuohy I. R., 1989, PASJ, 41, 427

onzález-Galán A., Kuulkers E., Kretschmar P., Larsson S., Postnov K., Kochetkova A., Finger M. H., 2012, A\&A, 537, A66

Hartman J. M. et al., 2008, ApJ, 675, 1468

Hinkle K. H., Fekel F. C., Joyce R. R., Wood P. R., Smith V. V., Lebzelter T., 2006, ApJ, 641, 479

Ibragimov A., Poutanen J., 2009, MNRAS, 400, 492

Iłkiewicz K., Mikołajewska J., Monard B., 2017, A\&A, 601, A105

Jahoda K., Markwardt C. B., Radeva Y., Rots A. H., Stark M. J., Swank J. H., Strohmayer T. E., Zhang W., 2006, ApJS, 163, 401

Lewin W. H. G., Ricker G. R., McClintock J. E., 1971, ApJ, 169, L17

Malacaria C., Mihara T., Santangelo A., Makishima K., Matsuoka M., Morii M., Sugizaki M., 2016, A\&A, 588, A100

Mony B. et al., 1991, A\&A, 247, 405

Nagase F., 1989, PASJ, 41, 1

Patruno A., 2010, ApJ, 722, 909

Patruno A., Watts A. L., 2012, preprint (arXiv:1206.2727)

Patruno A., Wijnands R., van der Klis M., 2009, ApJ, 698, L60

Patruno A., Alpar M. A., van der Klis M., van den Heuvel E. P. J., 2012, ApJ, 752, 33 
Pereira M. G., Braga J., Jablonski F., 1999, ApJ, 526, L105

Rea N., Stella L., Israel G. L., Matt G., Zane S., Segreto A., Oosterbroek T., Orlandini M., 2005, MNRAS, 364, 1229

Serim M. M., Şahiner Ş., ćerri-Serim D., Inam S. ć., Baykal A., 2017, MNRAS, 469, 2509

van den Eijnden J., Degenaar N., Russell T. D., Miller-Jones J. C. A., Wijnands R., Miller J. M., King A. L., Rupen M. P., 2018, MNRAS, 474, L91 van Straaten S., van der Klis M., Méndez M., 2003, ApJ, 596, 1155

Yoshida Y., Kitamoto S., Suzuki H., Hoshino A., Naik S., Jaisawal G. K., 2017, ApJ, 838, 30

This paper has been typeset from a $\mathrm{T}_{\mathrm{E}} \mathrm{X} / \mathrm{L} \mathrm{T} \mathrm{E} \mathrm{X}$ file prepared by the author. 